\title{
Comparaison des races Ile-de-France et Mérinos précoce en race pure et en croisement avec la race Boujaâd au Maroc
}

\author{
I. Boujenane ${ }^{1}$
}

\begin{abstract}
Mots-clés
Ovin - Ile-de-France - Mérinos précoce - Boujaâd - Reproduction Poids - Croisement - Maroc.
\end{abstract}

\begin{abstract}
Résumé
L'étude a porté sur 1145 performances de brebis et 1411 performances d'agneaux des races Ile-de-France (IF), Mérinos précoce (MP) et Boujaâd (BO). Les données ont été collectées durant huit saisons d'agnelage, allant de 1997-98 à 2004-05 dans un élevage privé. Les brebis IF et MP ont été accouplées avec des béliers de leurs races respectives, alors que les brebis BO ont été saillies par les béliers des trois races. La race de la brebis a eu un effet significatif sur la taille et le poids de portée à la naissance, mais pas sur la taille et le poids de portée à 70 jours (j). Les brebis IF et MP ont réalisé une taille et un poids de portée à la naissance supérieurs à la moyenne des performances des brebis $\mathrm{BO}$. Les différences ont été respectivement de 0,25 et 0,30 agneau, et de 1,45 et 1,22 kg. En outre, la race du bélier de lutte n'a pas eu d'effet significatif sur la taille de portée à la naissance des brebis $\mathrm{BO}$, mais elle a eu un effet significatif sur leur poids de portée à la naissance. Les brebis croisées avec les béliers IF et MP ont enregistré une performance moyenne de $0,48 \mathrm{~kg}$ supérieure à celle des brebis accouplées avec des béliers BO. Par ailleurs, le groupe génétique de l'agneau a eu un effet significatif sur les poids et la viabilité à $70 \mathrm{j}$. Les agneaux IF et MP ont réalisé les poids à la naissance les plus élevés, suivis par les agneaux croisés. Cependant, ces derniers ont réalisé des poids à 30 et 70 j plus élevés que ceux des agneaux des races parentales. Les agneaux IF ont enregistré une viabilité de 6 p. 100 inférieure à celle des agneaux MP et BO, et de 11 p. 100 plus faible que celle des agneaux croisés. L'effet hétérosis a été positif et élevé pour les poids à 30 et 70 j, et pour la viabilité à 70 j, mais négatif pour le poids à la naissance.
\end{abstract}

1. Département des productions animales, Institut agronomique et vétérinaire Hassan II, BP 6202, Rabat-Instituts, 10101 Rabat, Maroc

Tél./fax : +212 37776420 ; e-mail : i.boujenane@iav.ac.ma les élevages pratiquant le croisement industriel en béliers de races améliorées pures, certains élevages se sont spécialisés dans la sélection et la multiplication de ces races. Pour cela, ils ont suivi un contrôle de croissance et ont pratiqué la sélection selon les règles de la Commission nationale de sélection et de marquage. Il est donc nécessaire de connaître les performances des races Ile-deFrance et Mérinos précoce nées et élevées au Maroc afin de mieux orienter les éleveurs. L'objectif de cette étude a été de comparer les performances de reproduction et de croissance des races Ile-deFrance et Mérinos précoce en race pure et en croisement avec les brebis de race Boujaâd.

\section{MATERIEL ET METHODES}

Les données analysées étaient issues d'un élevage privé situé à $80 \mathrm{~km}$ au sud de Casablanca. Elles ont été collectées pendant huit campagnes d'agnelage, allant de 1997-98 à 2004-05. 


\section{Conduite du troupeau}

\section{Conduite de la reproduction}

Le troupeau de base était composé des races Ile-de-France (IF), Mérinos précoce (MP) et Boujaâd (BO). Il a été conduit au rythme d'un agnelage par an. Les brebis des races IF et MP ont été luttées par des béliers de leurs races respectives pour produire des agneaux de race pure. Celles de race BO ont été luttées par des béliers des trois races pour produire aussi bien les agneaux de race pure que les agneaux croisés (tableau I). Le nombre de béliers utilisés a varié d'une année à l'autre selon l'effectif de brebis disponibles. Les béliers ont généralement été remplacés après deux ou trois années de service. La lutte pratiquée était de type contrôlé, à raison de 30 brebis par bélier. Les lots de lutte étaient composés d'un mélange de brebis IF et BO ou de brebis MP et BO. La lutte principale a duré 60 jours et a eu lieu entre la mi-mai et la mi-juillet. Elle était habituellement suivie d'une lutte de rattrapage d'une durée d'un mois. Les agnelages se sont étalés sur une période de quatre mois, allant de début octobre jusqu'à fin janvier.

\section{Conduite alimentaire}

Les brebis étaient souvent en stabulation permanente. Elles recevaient une alimentation constituée de foin et d'un concentré à base d'orge. Parfois, elles étaient placées sur jachère pendant une partie de la journée. Les agneaux étaient en permanence en bergerie et ne sortaient jamais sur le parcours. Ils ont eu accès à un aliment complémentaire dès l'âge de 15 jours. Les agneaux mâles n'ont pas été castrés. En revanche, les agneaux mâles et femelles des races pures IF et MP destinés à la sélection ont subi une caudectomie.

\section{Conduite prophylactique}

Les brebis ont été vaccinées contre l'entérotoxémie un mois avant l'agnelage. Les agneaux l'ont été trois semaines après la naissance avec un rappel un mois plus tard. De plus, tous les animaux du troupeau ont subi un déparasitage interne et externe.

\section{Contrôles effectués}

A la naissance, les agneaux ont été identifiés par une boucle (tip tag) et la date de naissance, le groupe génétique, le sexe, le type de naissance et le numéro de la mère ont été enregistrés. Les agneaux ont été pesés à la naissance et une fois toutes les trois semaines jusqu'au sevrage qui a eu lieu vers l'âge de 70 jours (j).

\section{Variables étudiées}

Les caractères de reproduction étudiés ont été la taille de portée à la naissance (nombre total d'agneaux nés, vivants et morts), le poids de portée à la naissance (somme des poids à la naissance des agneaux de la brebis), la taille de portée à $70 \mathrm{j}$ (nombre des agneaux présents à $70 \mathrm{j}$ par brebis ayant agnelé) et le poids de portée à $70 \mathrm{j}$ (poids des agneaux présents à $70 \mathrm{j}$ par brebis ayant agnelé). Chez les agneaux, les caractères étudiés ont été les poids à la naissance, à $30 \mathrm{j}$ et à $70 \mathrm{j}$, ainsi que la viabilité entre la naissance et $70 \mathrm{j}$. Les poids à 30 et $70 \mathrm{j}$ ont été calculés par interpolation linéaire à partir des pesées qui ont eu lieu avant et après l'âge type considéré. Parfois, lorsque la pesée située après l'âge de 70 j n'était pas disponible, le poids à cet âge a été calculé par extrapolation linéaire.

\section{Analyses statistiques}

L'étude a porté sur 1145 performances de brebis et 1411 performances d'agneaux dont les données ont été collectées pendant les huit campagnes. Néanmoins, excepté la race IF dont les données sur les performances ont été disponibles durant toutes les campagnes, celles des autres groupes génétiques ne l'ont été qu'à certaines campagnes (tableau II). Par ailleurs, en raison de leur faible fréquence, les agneaux nés triples ont été éliminés de l'étude. Les données ont été analysées par la méthode des moindres carrés à l'aide de la procédure GLM (10).

Le modèle statistique utilisé pour analyser les performances des brebis a inclus la race du bélier de lutte (IF, MP et BO), la race de la brebis (IF, MP et BO) hiérarchisée dans la race du bélier et la campagne d'agnelage (1997-98, 1998-99 ... 2004-05). Les interactions de premier ordre se sont avérées non significatives $(\mathrm{P}>0,05)$ sur les caractères étudiés.

Les poids et la viabilité des agneaux ont été analysés en utilisant le modèle incluant le groupe génétique de l'agneau (BO, IF, MP, IF x BO et MP x BO), le sexe (mâle et femelle), la campagne de naissance (1997-98... 2004-05) et le type de naissance (simple et double) pour le poids à la naissance et la viabilité à $0-70 \mathrm{j}$ ou le type de naissance - élevage (simple - simple, double - double, et double - simple) pour les poids à 30 et $70 \mathrm{j}$. Les interactions du premier ordre ont été testées. Les interactions groupe génétique $\mathrm{x}$ type de naissance et type de naissance $\mathrm{x}$ campagne de naissance ont été significatives pour le poids à la naissance, et l'interaction type de naissance $\mathrm{x}$ campagne de naissance a été significative pour la viabilité $0-70 \mathrm{j}$.

Bien que les données sur les croisés réciproques n'aient pas été disponibles, les effets hétérosis individuel sur les poids et la viabilité des agneaux ont été estimés pour les croisements IF x BO et MP x BO comme la différence entre la moyenne des performances des agneaux croisés et la moyenne des performances des agneaux des races parentales.

\section{Tableau I}

Répartition des brebis et des agneaux selon la race de la brebis (mère) et la race du bélier de lutte (père)

\begin{tabular}{|c|c|c|c|c|c|c|c|c|}
\hline \multirow[t]{2}{*}{ Race de la brebis } & \multicolumn{3}{|c|}{ Race du bélier de lutte } & \multirow[t]{2}{*}{ Total } & \multicolumn{3}{|c|}{ Race du père de l'agneau } & \multirow[t]{2}{*}{ Total } \\
\hline & BO & IF & MP & & BO & IF & MP & \\
\hline $\mathrm{BO}$ & 154 & 233 & 188 & 575 & 161 & 254 & 211 & 626 \\
\hline IF & - & 474 & - & 474 & - & 647 & - & 647 \\
\hline MP & - & - & 96 & 96 & - & - & 138 & 138 \\
\hline Total & 154 & 707 & 284 & 1145 & 161 & 901 & 349 & 1411 \\
\hline
\end{tabular}

BO : Boujaâd ; IF : Ile-de-France ; MP : Mérinos précoce 
RESULTATS

\section{Performances de reproduction}

Les moyennes arithmétiques de l'ensemble des brebis pour la taille de portée à la naissance, la taille de portée à $70 \mathrm{j}$, le poids de portée à la naissance et le poids de portée à $70 \mathrm{j}$ ont été respectivement de $1,24,1,10,5,52 \mathrm{~kg}$ et $24,2 \mathrm{~kg}$ (tableau III).

La taille de portée à la naissance a varié de 1 à 3 chez les brebis $\mathrm{BO}$ et IF et de 1 à 2 chez les brebis MP. Les fréquences des portées simples, doubles et triples ont été respectivement de 90,4, 9,0 et 0,5 p. 100 chez les brebis BO, et de 63,1,36,7 et 0,2 p. $100 \mathrm{chez}$ les brebis IF. Les portées simples et doubles ont été respectivement de 56,3 et 43,8 p. 100 chez les brebis MP.

La race de la brebis a eu un effet significatif sur la taille de portée à la naissance. Les brebis MP ont réalisé une taille de portée à la naissance de 0,02 agneau plus élevée que celle des brebis IF, mais la différence n'a pas été significative $(\mathrm{P}>0,05)$. En revanche, les brebis $\mathrm{BO}$ ont enregistré une taille de portée moyenne à la naissance de 0,30 et 0,25 agneau de moins que celles respectivement des brebis MP et IF. En outre, la taille de portée à la naissance des brebis BO n'a pas été influencée par la race du bélier de lutte. Les différences entre les brebis saillies par les béliers des trois races ont été négligeables $(\mathrm{P}>0,05)$.

Le poids de portée à la naissance a été influencé par la race de la brebis. Il était presque similaire chez les brebis IF et MP, mais respectivement de 1,45 et 1,22 kg plus élevé que la moyenne des poids de portée des brebis BO. Par ailleurs, le poids de portée à la naissance des brebis $\mathrm{BO}$ a été différent selon la race du bélier de lutte. La différence n'a pas été significative entre les brebis saillies par les béliers des deux races à viande. En revanche, elle a été de $0,47 \mathrm{~kg}$ en faveur des brebis BO croisées avec les béliers de races améliorées par rapport à celles accouplées avec des béliers $\mathrm{BO}$.

\section{Tableau II}

Nombre de performances disponibles sur les brebis et les agneaux par groupe génétique et par campagne d'agnelage

\begin{tabular}{|c|c|c|c|c|c|c|c|c|c|c|}
\hline \multirow[t]{2}{*}{ Campagne } & \multicolumn{3}{|c|}{ Race de la brebis } & \multirow[t]{2}{*}{ Total } & \multicolumn{5}{|c|}{ Groupe génétique de l'agneau } & \multirow[t]{2}{*}{ Total } \\
\hline & IF & MP & BO & & IF & MP & BO & IF x BO & $\mathrm{MP} \times \mathrm{BO}$ & \\
\hline 1997-98 & 66 & - & 109 & 175 & 94 & - & 33 & 83 & - & 210 \\
\hline 1998-99 & 70 & - & 133 & 203 & 92 & - & - & 144 & - & 236 \\
\hline $1999-2000$ & 73 & - & 31 & 104 & 108 & - & 34 & - & - & 142 \\
\hline 2000-01 & 70 & - & 97 & 167 & 91 & - & - & - & 107 & 198 \\
\hline 2001-02 & 69 & - & 91 & 160 & 88 & - & 94 & - & - & 182 \\
\hline 2002-03 & 55 & 20 & 65 & 140 & 72 & 29 & - & - & 76 & 177 \\
\hline 2003-04 & 30 & 33 & 49 & 112 & 41 & 46 & - & 27 & 28 & 142 \\
\hline 2004-05 & 41 & 43 & - & 84 & 61 & 63 & - & - & - & 124 \\
\hline
\end{tabular}

BO : Boujaâd ; IF : Ile-de-France ; MP : Mérinos précoce

\section{Tableau III}

Moyennes des moindres carrés \pm erreurs-types des tailles de portée à la naissance et au sevrage, et des poids de portée à la naissance et au sevrage des brebis

\begin{tabular}{|c|c|c|c|c|c|}
\hline Source de variation & $\mathbf{n}$ & $\begin{array}{c}\text { Taille portée } \\
\text { naissance }\end{array}$ & $\begin{array}{l}\text { Poids portée } \\
\text { naissance (kg) }\end{array}$ & $\begin{array}{c}\text { Taille portée } \\
70 \text { j }\end{array}$ & $\begin{array}{c}\text { Poids portée } \\
70 \text { j (kg) }\end{array}$ \\
\hline Moyenne arithmétique & 1145 & 1,24 & 5,52 & 1,10 & 24,2 \\
\hline Coefficient de variation (\%) & & 35,1 & 30,4 & 43,5 & 43,4 \\
\hline $\begin{array}{l}\text { Race de la brebis : } \\
\text { race du bélier }\end{array}$ & & $* * *$ & $* * *$ & NS & NS \\
\hline IF : IF & 474 & $1,37^{a} \pm 0,02$ & $6,29^{a} \pm 0,07$ & $1,14 \pm 0,02$ & $25,1 \pm 0,48$ \\
\hline$M P: M P$ & 96 & $1,42^{a} \pm 0,05$ & $6,06^{a} \pm 0,17$ & $1,25 \pm 0,05$ & $25,0 \pm 1,18$ \\
\hline $\mathrm{BO}: \mathrm{BO}$ & 154 & $1,06^{b} \pm 0,04$ & $4,49^{b} \pm 0,15$ & $0,95 \pm 0,05$ & $18,6 \pm 1,05$ \\
\hline $\mathrm{BO}: \mathrm{IF}$ & 233 & $1,14^{b} \pm 0,04$ & $4,89^{\mathrm{c}} \pm 0,13$ & $1,08 \pm 0,04$ & $24,5 \pm 1,28$ \\
\hline $\mathrm{BO}: \mathrm{MP}$ & 188 & $1,16^{b} \pm 0,04$ & $5,06^{\mathrm{c}} \pm 0,14$ & $1,14 \pm 0,04$ & $27,3 \pm 0,94$ \\
\hline
\end{tabular}

*** $\mathrm{P}<0,001 ; \mathrm{NS}: \mathrm{P}>0,05$

IF : Ile-de-France ; MP : Mérinos précoce ; BO : Boujaâd

a,b,c Les moyennes ajustées d'une même colonne, suivies de lettres différentes, diffèrent $(\mathrm{P}<0,05)$ 
Bien que la taille et le poids de portée à 70 j aient été plus élevés chez les brebis IF et MP par rapport aux brebis BO, la race de la brebis n'a pas eu d'effet significatif $(\mathrm{P}>0,05)$. La différence de la taille de portée au sevrage entre les brebis des races améliorées pures et celles de race BO a été plus faible que la différence observée pour la taille de portée à la naissance. Par ailleurs, les brebis BO accouplées avec des béliers de la même race ont enregistré un poids de portée à $70 \mathrm{j}$ plus faible que celui des autres brebis. De même, les brebis BO croisées avec des béliers IF et MP ont réalisé des performances similaires à celles des brebis des races améliorées pures.

\section{Performances de croissance et de viabilité des agneaux}

Les moyennes arithmétiques des poids à la naissance, à $30 \mathrm{j}$ et à $70 \mathrm{j}$ de tous les agneaux ont été respectivement de 4,46, 11,2 et 22,0 kg. La viabilité à 0-70 j a été en moyenne de 89 p. 100 (tableau IV).

Le groupe génétique de l'agneau a eu un effet significatif sur les poids étudiés et sur la viabilité $0-70 \mathrm{j}$. Les poids à la naissance, à 30 et à $70 \mathrm{j}$ des agneaux IF ont été respectivement de 0,28, 0,87 et $1,20 \mathrm{~kg}$ plus élevés que ceux des agneaux MP et de 0,71, 1,90 et $4,2 \mathrm{~kg}$ supérieurs à ceux des agneaux $\mathrm{BO}$. Les poids à la naissance des agneaux croisés ont été intermédiaires par rapport à ceux des races parentales. En revanche, leurs poids à 30 et $70 \mathrm{j}$ ont été plus élevés que ceux des agneaux des races parentales.

Le groupe génétique de l'agneau a eu un effet significatif sur la viabilité entre la naissance et 70 jours. Les agneaux IF ont enregistré la viabilité la plus faible (83 p. 100), les agneaux croisées la viabilité la plus élevée (94 p. 100), et les agneaux MP et BO une viabilité intermédiaire (88 p. 100).

Les croisements entre les races IF et BO, d'une part, et MP et BO, d'autre part, ont engendré un effet hétérosis sur les poids et la viabilité des agneaux. Les pourcentages d'hétérosis pour les poids à la naissance, à $30 \mathrm{j}$, à $70 \mathrm{j}$ et la viabilité à $70 \mathrm{j}$ ont été respectivement de $-6,0,14,3,13,2$ et 8,23 p. 100 pour le croisement entre les races IF et BO. Ils ont été de - 1,46, 12,7, 15,1 et 8,24 p. 100 pour le croisement entre les races MP et BO. Ces effets d'hétérosis ont été significatifs, sauf pour le poids à la naissance du croisement MP x BO.

\section{DISCUSSION}

Les tailles de portée à la naissance des brebis des races IF et MP ont été presque similaires. De même, ces races n'ont apparemment pas connu de difficultés d'adaptation, puisque leurs performances n'ont pas été très différentes de celles réalisées en France qui sont respectivement de 1,56 et 1,41 (1). Les brebis BO ont enregistré une taille de portée à la naissance intermédiaire, comparée à celle de 1,04 obtenue chez les éleveurs (4) et celle de 1,29 réalisée en station (5). Par ailleurs, l'absence de l'effet de la race du bélier sur la taille de portée des brebis $\mathrm{BO}$ a été conforme aux résultats rapportés par d'autres auteurs $(2,6,7,8)$ qui ont montré que ce caractère était lié à la brebis et qu'il était peu ou n'était pas influencé par la race du bélier.

Le poids de la portée à la naissance a été élevé chez les brebis IF et MP, faible chez les brebis BO saillies par les béliers de la même race et intermédiaire chez celles croisées avec des béliers IF et MP. Cette supériorité des brebis des races améliorées peut être expliquée à la fois par leur taille de portée à la naissance et par le poids à la naissance des agneaux qui sont élevés. De même, la supériorité des brebis BO croisées avec des béliers de races à viande par rapport à celles saillies par des béliers $\mathrm{BO}$ peut être due au poids à la naissance élevé des agneaux croisés.

La taille et le poids à $70 \mathrm{j}$ n'ont pas été influencés par la race de la brebis. En effet, le taux de mortalité élevé enregistré chez les agneaux issus des brebis IF et MP a très fortement diminué leur taille de portée à $70 \mathrm{j}$, réduisant ainsi l'avantage qu'elles avaient sur les brebis BO. Ce résultat n'est pas conforme à ceux d'El Fadili et Leroy (6), et de Boujenane et Kansari (3) qui ont rapporté des différences significatives entre les performances des brebis de différents groupes génétiques.

Le groupe génétique a un effet significatif sur les poids des agneaux. Les poids à la naissance réalisés semblaient normaux puisque les agneaux des races améliorées étaient les plus lourds, ceux de race pure BO les plus légers et ceux des agneaux croisés intermédiaires. En revanche, la supériorité des poids à 30 et 70 j des agneaux croisés par rapport aux agneaux IF et MP n'était pas très attendue, mais peut s'expliquer par le fait que la race pure $\mathrm{BO}$, conduite de façon

\section{Tableau IV}

Moyennes des moindres carrés \pm erreurs-types des poids à la naissance, à 30 et à 70 jours, et de la viabilité des agneaux

\begin{tabular}{|c|c|c|c|c|c|c|c|c|}
\hline $\begin{array}{l}\text { Source de } \\
\text { variation }\end{array}$ & $\mathbf{n}$ & $\begin{array}{c}\text { Poids à la } \\
\text { naissance (kg) }\end{array}$ & $\mathbf{n}$ & $\begin{array}{l}\text { Poids à } \\
30 \text { j (kg) }\end{array}$ & $\mathbf{n}$ & $\begin{array}{l}\text { Poids à } \\
70 \text { j (kg) }\end{array}$ & $\mathbf{n}$ & $\begin{array}{l}\text { Viabilité à } \\
0-70 \text { j (\%) }\end{array}$ \\
\hline Moyenne arithmétique & 1411 & 4,46 & 1100 & 11,2 & 1062 & 22,0 & 1411 & 89 \\
\hline Coefficient de variation (\%) & & 18,9 & & 27,6 & & 26,2 & & 35,3 \\
\hline Groupe génétique de l'agneau ${ }^{1}$ & & $* * *$ & & $* * *$ & & $* * *$ & & $* * *$ \\
\hline IF & 647 & $4,62^{\mathrm{a}} \pm 0,03$ & 551 & $10,7^{\mathrm{a}} \pm 0,15$ & 522 & $21,6^{a} \pm 0,32$ & 647 & $83^{a} \pm 1,2$ \\
\hline MP & 138 & $4,34^{b} \pm 0,07$ & 123 & $9,83^{b} \pm 0,28$ & 118 & $20,4^{b} \pm 0,58$ & 138 & $89^{a b} \pm 3,0$ \\
\hline BO & 161 & $3,91^{\mathrm{c}} \pm 0,10$ & 139 & $8,80^{\mathrm{c}} \pm 0,29$ & 139 & $17,4^{\mathrm{C}} \pm 0,59$ & 161 & $88^{a b} \pm 3,1$ \\
\hline IF x BO & 254 & $4,01^{c} \pm 0,06$ & 89 & $11,1^{\mathrm{a}} \pm 0,34$ & 89 & $22,1^{\mathrm{a}} \pm 0,69$ & 254 & $92^{b} \pm 2,6$ \\
\hline $\mathrm{MP} \times \mathrm{BO}$ & 211 & $4,06^{c} \pm 0,06$ & 198 & $10,5^{a b} \pm 0,26$ & 194 & $21,8^{a} \pm 0,53$ & 211 & $96^{b} \pm 2,7$ \\
\hline
\end{tabular}

$* * * \mathrm{P}<0,001$

IF : Ile-de-France ; MP : Mérinos précoce ; BO : Boujaâd

${ }^{1}$ Pour les agneaux croisés, la race du père est placée en premier

a,b,c Les moyennes ajustées d'une même colonne, suivies de lettres différentes, diffèrent $(\mathrm{P}<0,05)$ 
intensive, a enregistré des performances qui se rapprochent de celles des races améliorées (5). La comparaison des poids des agneaux de différents groupes génétiques a montré que ceux de race pure Lacaune à viande réalisent un poids à la naissance élevé, mais ils sont rattrapés à 30 et $70 \mathrm{j}$ par ceux de certains autres groupes génétiques (3). Par ailleurs, les agneaux croisés ont enregistré un effet hétérosis négatif pour le poids à la naissance, et positif et élevé pour les poids à 30 et $70 \mathrm{j}$. L'hétérosis observée pour ces deux derniers poids a été identique pour les deux croisements et supérieure à la valeur rapportée pour le poids au sevrage (9).

La viabilité entre la naissance et $70 \mathrm{j}$ a été significativement influencée par le groupe génétique de l'agneau. En effet, les agneaux croisés ont enregistré une viabilité plus élevée que celles des agneaux des races pures IF, MP et BO. La faible viabilité des agneaux IF peut être expliquée par leur sensibilité plus élevée aux problèmes sanitaires et aux conditions climatiques difficiles par rapport aux agneaux MP qui ont relativement fait preuve d'une certaine adaptation. Elle peut être également expliquée par un mode de conduite qui n'a pas été toujours très adéquat. Boujenane et Kansari (3) ont constaté que parmi les huit groupes génétiques étudiés, les agneaux croisés ont réalisé la viabilité la plus élevée et les agneaux de race pure Lacaune à viande ont enregistré la viabilité la plus faible. La viabilité élevée des agneaux croisés peut être due à une bonne adaptation de ce groupe génétique aux conditions d'élevage marocain, et à l'effet hétérosis positif et supérieur à la valeur de 9,8 p. 100 rapportée par Nitter (9).

\section{BIBLIOGRAPHIE}

1. BODIN L., ELSEN J.M., 1989. Variability of litter size of French sheep breeds following natural or induced ovulation. Anim. Prod., 48 : 535-541.

2. BOUJENANE I., BERRADA D., MIHI S., JAMAI M., 1998. Reproductive performance of ewes and preweaning growth of lambs from three native Moroccan breeds mated to rams from Moroccan and improved breeds. Small Rumin. Res., 27: 203-208.

3. BOUJENANE I., KANSARI J., 2002. Lamb production and its components from purebred and crossbred mating types. Small Ruminant Res., 43: 115-120.

4. Chentouf M., BOUlanOUAR B., 2000. Conduite de la reproduction des ovins dans une zone semi-aride du Maroc. EAAP Publ., $94: 255-259$.

5. CHIKHI A., BOUJENANE I., 2003. Performances de reproduction et de production des ovins de race Boujaâd au Maroc. Revue Elev. Méd. vét. Pays trop., $\mathbf{5 6}: 83-88$.

\section{CONCLUSION}

Cette étude a permis d'avoir une idée sur les performances des races pures IF et MP nées et élevées au Maroc. Elle a montré que ces deux races sont presque identiques du point de vue de la reproduction, légèrement différentes du point de vue de la croissance, avec un léger avantage pour la race IF, et différentes pour ce qui est de la viabilité des agneaux, avec une supériorité très nette de la race MP. Par ailleurs, les performances des races IF et MP ont été supérieures à celles des brebis $\mathrm{BO}$ utilisées en race pure. En revanche, les brebis IF et MP ont enregistré des productivités à $70 \mathrm{j}$ identiques à celles des brebis BO utilisées en croisement, et les agneaux améliorés ont réalisé des poids à 30 et 70 j légèrement inférieurs à ceux des croisés IF x BO et MP x BO. Il semblerait que dans les conditions marocaines, les performances des races IF et MP soient similaires en races pures ou en croisement avec la race $\mathrm{BO}$.

\section{Remerciements}

L'auteur tient à remercier M. R. Mourabit (propriétaire) et M. A. Daim (technicien de l'Anoc) pour avoir mis les données de cet élevage à sa disposition, et M. A. Marouane pour leur saisie.

6. EL FADILI M., LEROY P.L., 2000. Comparaison de trois races de croisement terminal pour la production des agneaux croisés de boucherie au Maroc. Ann. Méd. vét., 145 : 85-92.

7. LEYMASTER K.A., SMITH G.M., 1981. Columbia and Suffolk terminal sire breed effects. J. Anim. Sci., 53: 1225-1235.

8. NITTER G., 1975. Results of a crossbreeding experiment with sheep for different systems of fat lamb production. I. Reproductive traits. Livest. Prod. Sci., 2: 167-177.

9. NITTER G., 1978. Breed utilization for meat production in sheep Anim. Breed. Abstr., 46: 131-143.

10. SAS/STAT, 1996. User's Guide, vers. 6.12. Cary, NC, USA, SAS Institute.

Reçu le 07.07.2005, accepté le 20.02.2006 


\section{Summary}

Boujenane I. Comparison of Ile-de-France and Merino Precoce Breeds as Pure Breeds and in Crossbreeding with the Boujaâd Breed in Morocco

The study concerned 1145 performances of ewes and 1411 performances of lambs of Ile-de-France (IF), Merino precoce (MP) and Boujaâd (BO) breeds. The data were collected during eight lambing seasons, from 1997-98 to 2004-05 in a private farm. IF and MP ewes were mated to rams of their corresponding breeds, whereas $\mathrm{BO}$ ewes were mated to rams of the three breeds. The ewe breed had a significant effect on the litter size and litter weight at birth, but not on the litter size and litter weight at 70 days (d). IF and MP ewes had a litter size and a litter weight at birth higher than the corresponding performance average of $\mathrm{BO}$ ewes. The differences were 0.25 and 0.30 lamb, and 1.45 and $1.22 \mathrm{~kg}$, respectively. Moreover, the mating ram breed did not have a significant effect on the litter size at birth of BO ewes, but it had a significant effect on the litter weight at birth. The performance of ewes crossed with IF and MP rams was on average $0.48 \mathrm{~kg}$ higher than that of ewes mated with BO rams. In addition, the lamb genetic group had a significant effect on the lamb weight and viability at $70 \mathrm{~d}$. IF and MP lambs had the highest birth weights, followed by crossbred lambs. However, the weights at 30 and $70 \mathrm{~d}$ of the latter were higher than those of the parental breeds. IF lambs' viability was $6 \%$ lower than that of MP and BO lambs, and $11 \%$ lower than that of crossbred lambs. The heterosis effect was positive and high for weights at 30 and $70 \mathrm{~d}$, and for survival at $70 \mathrm{~d}$, but negative for birth weights.

Keywords: Sheep - Ile-de-France - Merino precoce Boujaâd - Reproduction - Weight - Crossbreeding - Morocco.

\section{Resumen}

Boujenane I. Comparación de razas Ile-de-France y Merinos precoz en raza pura y en cruce con la raza Boujaâd de Marruecos

El estudio se llevó a cabo sobre 1145 rendimientos de ovejas y 1411 rendimientos de corderos de las razas Ile-de-France (IF), Merinos precoz (MP) y Boujaâd (BO). Estos datos se recolectaron durante ocho estaciones de parto, de 1997-98 a 200405 en un establecimiento privado. Las ovejas IF y MP se acoplaron con corderos de las razas respectivas, mientras que las ovejas BO fueron montadas por corderos de las tres razas. La raza de la oveja tuvo un efecto significativo sobre el tamaño y el peso de la camada al nacimiento, pero no sobre el tamaño y el peso de la camada a 70 días (d). Las ovejas IF y MP presentaron un tamaño y un peso de camada al nacimiento superiores al promedio de los rendimientos de las ovejas BO. Las diferencias fueron de 0,25 y 0,30 por cordero respectivamente y de 1,45 y $1,22 \mathrm{~kg}$. Por otro lado, la raza del carnero de lucha no tuvo efecto significativo sobre el tamaño de la camada al nacimiento de las ovejas $\mathrm{BO}$, pero sí un efecto significativo sobre el peso de la camada al nacimiento. Las ovejas cruzadas con los carneros IF y MP registraron un rendimiento promedio 0,48 kg superior al de las ovejas acopladas con corderos BO. Por otro lado, el grupo genético del cordero tuvo un efecto significativo sobre los pesos y la viabilidad a $70 \mathrm{~d}$. Los corderos IF y MP presentaron los pesos al nacimiento más elevados, seguidos por los corderos cruzados. Sin embargo, estos últimos presentaron pesos a 30 a 70 d más altos que los de los corderos de razas puras. Por otro lado, los corderos IF mostraron una viabilidad $6 \%$ inferior a la de los corderos MP y BO y $11 \%$ inferior a la de los corderos cruzados. El efecto heterosis fue positivo y aumentó los pesos a 30 y 70 d y la viabilidad a $70 \mathrm{~d}$, pero negativo para el peso al nacimiento.

Palabras clave: Ovino - Ile-de-France - Merino precoce Boujaâd - Reproducción - Peso - Cruzamiento - Marruecos. 